\title{
Impact of quantized vibrations on the efficiency of interfacial charge separation in photovoltaic devices
}

\author{
Soumya Bera, ${ }^{1}$ Nicolas Gheeraert,${ }^{1}$ Simone Fratini, ${ }^{1}$ Sergio Ciuchi, ${ }^{2}$ and Serge Florens ${ }^{1}$ \\ ${ }^{1}$ Institut Néel, CNRS and Université Grenoble Alpes, F-38042 Grenoble, France \\ ${ }^{2}$ Dipartimento di Scienze Fisiche e Chimiche, Università dell'Aquila, \\ CNISM and Istituto Sistemi Complessi CNR, via Vetoio, I-67010 Coppito-L'Aquila, Italy
}

(Dated: 7th June 2018)

\begin{abstract}
We demonstrate that charge separation at donor-acceptor interfaces is a complex process that is controlled by the combined action of Coulomb binding for electron-hole pairs and partial relaxation due to quantized phonons. A joint electron-vibration quantum dynamical study reveals that high energy vibrations sensitively tune the charge transfer probability as a function of time and injection energy, due to polaron formation. These results have bearings for the optimization of energy transfer both in organic and quantum dot photovoltaics, as well as in biological light harvesting complexes.
\end{abstract}

Introduction. The dissociation of neutral excitons into separate charge carriers is a key physical process happening in various light harvesting devices, such as organic materials [1, quantum dot assemblies [2, 3, and photosynthesis complexes [4. In the case of organic photovoltaics which is our main focus here, an interface is created between a disordered polymer (donor), and a fullerene derivative (acceptor). Because of poor dielectric screening and the narrow bandwidth of the organic acceptor, an electron that experiences energetic losses during its transfer at the interface may be tightly bound to the hole it has left behind. Such an interfacial exciton displays a binding energy which is typically an order of magnitude larger than the thermal energy $k_{B} T$ at room temperature. A highly debated issue $5-9$ is therefore whether the exciton created by absorption of a photon will eventually relax to the lowest available energy bound state across the interface, therefore precluding the coherent migration of the carriers to the collecting electrode, or if it can separate at sufficiently long distances prior to relaxation, enabling photocurrent generation. These two extreme views are presently difficult to reconcile, but both should certainly be at play in the microscopic mechanisms that ultimately control the efficiency of energy transfer in these systems.

Our aim in this Letter is to study microscopically the role of quantized phonons - i.e. discrete molecular vibrations whose characteristic energy is larger than $k_{B} T$ - in the charge transfer process. As opposed to low energy baths (due to intermolecular phonons or solvents), which lead to damping processes and final equilibration on long time scales, high energy intramolecular vibrations correspond to fast time scales in the ten-femtosecond range, comparable to the excitonic dissociation process. Such quantized modes are strongly coupled to the electronic orbitals, both in photosynthetic systems [10] and in fullerenes, as revealed e.g. by ab initio theoretical calculations [11, 12], transport [13] and photoemission experiments [14. More importantly, their relevance to the charge transfer through organic interfaces has been pinpointed very recently by ultrafast spectroscopic techniques
[15, 16. All these observations indicate that high-energy vibrations should be included in any consistent model for organic photovoltaic devices. We unveil here that, by coherently dressing the charge carriers, quantized phonons sensitively control the charge transfer probability, and can be hugely detrimental to free carrier generation in the presence of interfacial Coulomb interaction between hole and electron species.

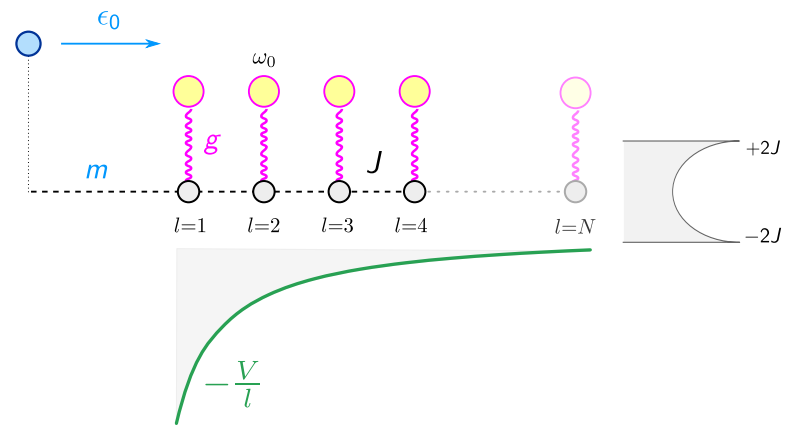

Figure 1: Schematic diagram of the electron-vibration chain model that it used the describe the dynamics at the donoracceptor interface Eq. (1). $\epsilon_{0}$ is the electronic energy after photoexcitation on the donor side, and the green curve represents the attractive Coulomb potential from the hole, that we suppose to be localized at site $l=0$. The density of states of the non-interacting chain is shown on the right.

Model. We use the following Hamiltonian to describe the quantum-mechanical exciton charge transfer process in a molecular chain of length $N$ (depicted on Fig. 1):

$$
\begin{aligned}
\mathcal{H} & =\omega_{0} \sum_{l=1}^{N} a_{l}^{\dagger} a_{l}+g \sum_{l=1}^{N} c_{l}^{\dagger} c_{l}\left(a_{l}+a_{l}^{\dagger}\right)-m\left(c_{0}^{\dagger} c_{1}+c_{1}^{\dagger} c_{0}\right) \\
& +\epsilon_{0} c_{0}^{\dagger} c_{0}-\sum_{l=1}^{N} \frac{V}{l} c_{l}^{\dagger} c_{l}-J \sum_{l=1}^{N-1}\left(c_{l}^{\dagger} c_{l+1}+c_{l+1}^{\dagger} c_{l}\right) \cdot(1)
\end{aligned}
$$

Here $a_{l}^{\dagger}$ and $c_{l}^{\dagger}$ are respectively the phonon and electron creation operators on site $l, \omega_{0}$ is the energy of the relevant molecular vibration, $g$ is the electron-phonon coupling 
constant, $m$ is the tunneling amplitude between the donor (site $l=0$ ) and the first site of the tight binding chain describing the acceptor, $\epsilon_{0}$ is the energy of the incoming electron (related to the difference between the donor and acceptor band offset and the exciton binding energy), $V$ sets the typical Coulomb potential binding the electronhole pair, and $J$ is the hopping amplitude within the chain. In the following paragraphs we shall use the dimensionless Huang-Rhys parameter $\alpha^{2}=\left(g / \omega_{0}\right)^{2}$ - i.e. the ratio between the molecular reorganization energy $\lambda$ and the vibrational energy $\omega_{0}$ - to characterize the strength of the electron-phonon interaction, as appropriate in the non-adiabatic regime $\omega_{0} \gtrsim J[17,18$.

We consider here simultaneously the combined effect of charge transfer processes near the interface and the delocalized motion away from it, in contrast to previous studies focusing either on the former [19, 20] or the latter [8, 21 23 issue. First, we will not model the hole dynamics on the donor side, assuming that it is localized on site $l=0$. The effects of hole diffusion, that could be easily described by extending Hamiltonian Eq. (1), typically amounts to a reduction of the effective Coulomb binding. Second, a tight-binding model on a one-dimensional chain is used to describe a complex bulk molecular heterostructure. The three-dimensional nature of the system and the realistic molecular orbitals are expected to lead to mainly quantitative changes in the bandstructure, which are currently the subject of theoretical investigation [8, 20, 24 27. Third, we assume a single intramolecular mode of vibration, as vindicated by the few high frequency discrete modes of fullerene molecules [11, 12, 14] (in the narrow range $0.18-0.2 \mathrm{eV}$ ) that account for the majority of the electron-phonon coupling. The electron dynamics considered here concerns the fast electron unbinding from the Coulomb well, occurring on the 10 to 100 femtosecond time scale [15, 16. We thus neglect the impact of weakly coupled low frequency phonon modes [10, 11, 13, 28, as these will lead to final equilibration at longer relaxation times than relevant for the exciton dissociation process. Fourth, we take a pure Coulomb law for the electron-hole binding potential, but any other type of interaction should not drastically affect our general results (see Refs. 25, 29 for a more microscopic approach). We also choose to ignore disorder-induced localization effects, 21, 23, 28, since a localization mechanism is already provided by the interfacial binding potential. Finally, we study the dynamical evolution of the system at zero temperature, which is justified because both electronic and vibrational energy scales are much larger than $k_{B} T$ [30].

Theoretical approach. Model Eq. (1) and variants have been the topic of several recent theoretical studies focusing either on detailed aspects of the exciton dissociation at the interface [19, 20] or on dynamics of charge separation on the acceptor chain $[8,21,22,28$. Our goal is to provide a joint quantum dynamical description for the electron and vibrational modes on the chain - whereas vibrational dynamics is often approximated by a classical evolution or by an effective decay rate on the electron motion and to explore the resulting microscopic phenomena in a broad range of relevant parameters. The approach that we propose is the use of Dirac-Frenkel's time-dependent variational principle [31, which has been applied with success to study ground state and dynamical properties of a wide class of open quantum systems 32 35]. We will start with the following wavefunction Ansatz:

$$
|\Psi(t)\rangle=\sum_{l=0}^{N} A_{l}(t) c_{l}^{\dagger} e^{f_{l}(t) a_{l}^{\dagger}-f_{l}^{\star}(t) a_{l}}|0\rangle .
$$

Here $A_{l}(t)$ corresponds to the local electron complex probability amplitude at site $l$ and time $t$, while the complex parameter $f_{l}(t)$ describes a coherent state of the phonon mode $a_{l}(|0\rangle$ is the full fermionic and bosonic vacuum). We emphasize that the dynamics in the wavefunction Eq. (2) is restricted to a single electron propagating along the whole chain, hence reducing drastically the fermionic Hilbert space to the size $N+1$. However, the full electronphonon problem is non-trivial, because for finite values of $\alpha^{2}$, several Fock states have to be considered per site, making the total number of states exponentially large. An important rationale to vindicate Ansatz Eq. (2) is that it encompasses several known limits. First, in the absence of phonons $\left(\alpha^{2}=0\right)$, the exact electron dynamics is obtained in the presence of any binding potential (or even disorder). Second, when charge hopping is turned off $(J=0)$, local phonons experience a displacement $f_{l}$ depending on whether a charge is present or not, an effect that is exactly contained in our wavefunction. A crucial aspect of the Ansatz is that it correctly captures the so-called non-adiabatic regime [17, 18], whereupon the phonon frequency $\omega_{0}$ is not small compared to the tunneling energy, which is the case for the intra-molecular vibrations considered here.

We describe the time evolution of wave-function Eq. (2) by constructing the following Lagrangian: $(\hbar=1$ in what follows):

$$
\begin{aligned}
\mathcal{L}= & \left\langle\Psi(t)\left|\frac{i}{2} \overleftrightarrow{\partial_{t}}-\mathcal{H}\right| \Psi(t)\right\rangle \\
= & \frac{i}{2} \sum_{l=0}^{N}\left|A_{l}\right|^{2}\left[f_{l}^{\star} \dot{f}_{l}-\dot{f}_{l}^{\star} f_{l}\right]+\frac{i}{2} \sum_{l=0}^{N}\left[A_{l}^{\star} \dot{A}_{l}-\dot{A}_{l}^{\star} A_{l}\right] \\
& +\sum_{l=1}^{N}\left|A_{l}\right|^{2}\left[\omega_{0}\left|f_{l}\right|^{2}+g\left(f_{l}+f_{l}^{\star}\right)+\epsilon_{l}\right] \\
& -\sum_{l=1}^{N} J_{l}\left[A_{l}^{\star} A_{l+1}+A_{l+1}^{\star} A_{l}\right] e^{-\left|f_{l}\right|^{2} / 2-\left|f_{l+1}\right|^{2} / 2},(4)
\end{aligned}
$$

where we use the compact notation $\overleftrightarrow{\partial_{t}}=\vec{\partial} / \partial t-\overleftarrow{\partial} / \partial t$ $\dot{A}_{l}=\partial A_{l} / \partial t, \dot{f}_{l}=\partial f_{l} / \partial t, \epsilon_{l}=\epsilon_{0} \delta_{l, 0}-\frac{V}{l}\left(1-\delta_{l, 0}\right)$, and $J_{l}=m \delta_{l, 0}+J\left(1-\delta_{l, 0}\right)$. The full dynamical evolution can 

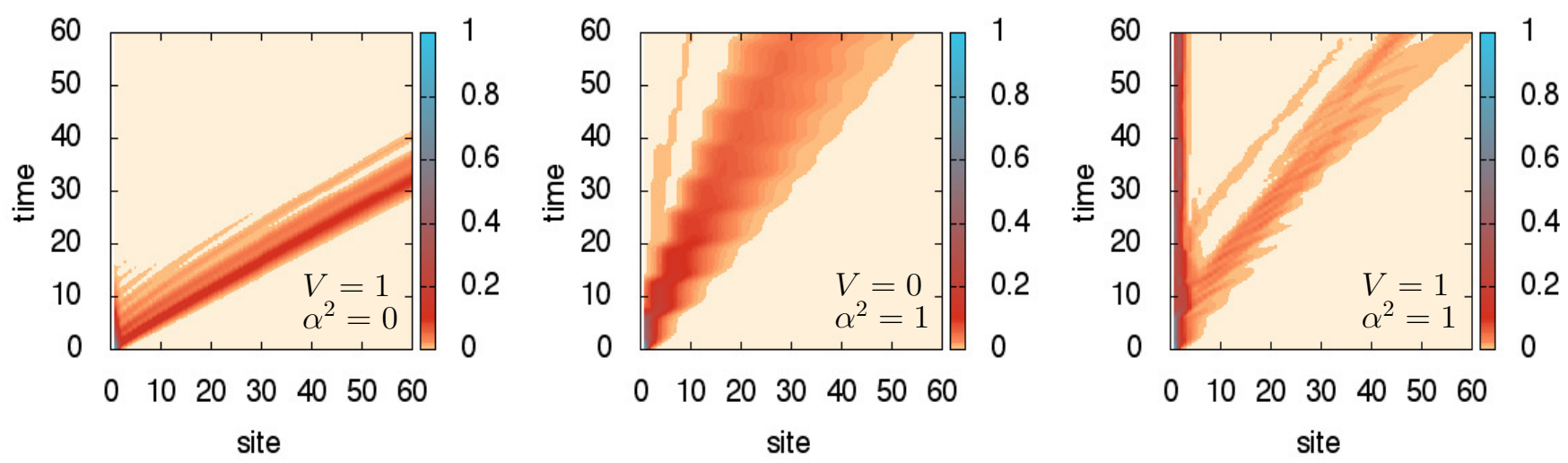

Figure 2: (color online) Flow of electron density $\left|A_{l}(t)\right|^{2}$ at each site $l$ as a function of time $t$ (in units of $1 / J \simeq 20 \mathrm{fs}$ ), for an injection energy $\epsilon_{0}=0.1$ that is taken within the available band of delocalized states. Three different dynamics are performed: left panel, Coulomb confinement only $\left(\alpha^{2}=0.0, V=1.0\right)$; middle panel, electron-phonon interaction only $\left(\alpha^{2}=1.0, V=0.0\right)$; right panel, both interaction and confinement $\left(\alpha^{2}=1.0, V=1.0\right)$. Only the latter case shows prominent localized states near the interface, that are however superposed with out-going wavepackets, giving a reduced but finite yield.

then be obtained from Hamilton-Jacobi equations:

$$
\begin{aligned}
\frac{\mathrm{d}}{\mathrm{d} t} \frac{\partial \mathcal{L}}{\partial \dot{f}_{i}^{\star}} & =\frac{\partial \mathcal{L}}{\partial f_{i}^{\star}}, \\
\frac{\mathrm{d}}{\mathrm{d} t} \frac{\partial \mathcal{L}}{\partial \dot{A}_{i}^{\star}} & =\frac{\partial \mathcal{L}}{\partial A_{i}^{\star}},
\end{aligned}
$$

which read explicitly as:

$$
\begin{aligned}
i \dot{f}_{l}= & \frac{J_{l} f_{l}}{A_{l}} e^{-\left|f_{l}\right|^{2} / 2}\left[A_{l+1} e^{-\left|f_{l+1}\right|^{2} / 2}+A_{l-1} e^{-\left|f_{l-1}\right|^{2} / 2}\right] \\
& +\omega_{0} f_{l}+g \equiv F_{l}, \\
i \dot{A}_{l}= & -\frac{A_{l}}{2}\left[f_{l} F_{l}^{\star}+f_{l}^{\star} F_{l}\right]+A_{l}\left[\omega_{0}\left|f_{l}\right|^{2}+g\left(f_{l}+f_{l}^{\star}\right)+\epsilon_{l}\right] \\
- & J_{l} e^{-\left|f_{l}\right|^{2} / 2}\left[A_{l+1} e^{-\left|f_{l+1}\right|^{2} / 2}+A_{l-1} e^{-\left|f_{l-1}\right|^{2} / 2}\right],
\end{aligned}
$$

where $f_{0}=0$ is enforced since we do not include phonons on the donor site. Note that the local displacement of the phonon $X_{l}(t)=\left\langle\Psi(t)\left|a_{l}^{\dagger}+a_{l}\right| \Psi(t)\right\rangle=$ $\left|A_{l}(t)\right|^{2}\left[f_{l}^{\star}(t)+f_{l}(t)\right]$ in the present notation. In the limit of isolated molecular sites $(J=0)$, the electron density is time-independent, and we find the physically correct vibrational dynamics: $\dot{P}_{l}=\omega_{0} X_{l}+2 g\left|A_{l}\right|^{2}$, with $P_{l}$ the conjugate momentum to $X_{l}$. The closed set of dynamical equations (7)-(8) can be efficiently and accurately solved using a fourth-order Runge-Kutta algorithm. Typically, a time step of $\delta t=0.01$ in the natural units ensures good convergence. Of particular importance to check the quality of the numerical solution is to monitor the norm $N=\langle\Psi(t) \mid \Psi(t)\rangle$ and the average energy $E=\langle\Psi(t)|\mathcal{H}| \Psi(t)\rangle$, and verify that they do not evolve with time, as these are exactly conserved quantities during the dynamics (as can be easily proven from the previous equations).

Results. Our initial state consists of an electron injected on the donor site $l=0$ with excess energy $\epsilon_{0}$, without any lattice distorsion on the acceptor side, namely $|\Psi(t=0)\rangle=c_{0}^{\dagger}|0\rangle$. We set the basic energy unit to be the hopping parameter $J=1$. We take the donor to acceptor tunneling rate $m=0.5$, having checked that our results do not depend much on this quantity as long as $m \lesssim 0.5$. We also fix the vibration frequency $\omega_{0}=1$, that we take in the realistic experimental range, but again the precise value is not very important as long as one remains in the non-adiabatic regime. We leave free the other three parameters i.e. the injection energy $\epsilon_{0}$, the electron-phonon vibronic coupling $\alpha^{2}$ and the Coulomb strength $V$.

We start by investigating the time-evolution of the wavefunction along the chain as time evolves. On Fig. 2 we consider the dynamics of the electron density $\left\langle\Psi(t)\left|c_{l}^{\dagger} c_{l}\right| \Psi(t)\right\rangle=\left|A_{l}(t)\right|^{2}$. The leftmost panel considers the sole effect of Coulomb confinement $\left(\alpha^{2}=0.0\right.$, $V=1.0$ ), and our results here are numerically exact, by construction of Ansatz Eq. (2). We find that the main contribution is in the form of pulses of ballistic wavepackets emitted from the interface. This bevavior is readily explained by the undamped oscillatory motion of the electron in the confinement zone, which leads to periodic emission of electron probability due to resonant scattering by the external potential. Clearly, the probability weight near the interface is progressively decaying with time, and the particle is fully delocalized, a picture often proposed in the literature [8, 21, 23], that however drastically overestimates the energy transfer efficiency of realistic devices. When the electron-phonon interaction is switched on, but without binding potential (middle panel, with $\alpha^{2}=1.0, V=0.0$ ), two striking effects arise due to the dressing of electronic states by phonons (polaron formation). First, for most values of the injection energy (here shown $\epsilon_{0}=0.1$ ), the beam is dominated by a fast 
ballistic component, albeit with a strong renormalization and dispersion of velocities. Second, temporal oscillations of period $2 \pi / \omega_{0}$ of the electron transfer probability appear, clearly associated to vibronic coupling.

The right panel in Fig. 2 shows the most interesting case where both interaction and confinement are taken into account $\left(\alpha^{2}=1.0, V=1.0\right)$. While the presence of molecular vibrations or Coulomb binding taken alone do not prevent the migration of electrons to the electrodes, their combined effect provides strong interfacial relaxation, leading to prominent localized states near the interface. The observed collapse of the exciton wavefunction in proximity of the interface drastically affects the transmission, which yet remains finite, as is seen from the existence of a ballistic beam of electrons with reduced amplitude (compared to the left and middle panels). As seen in Fig. 2, charge transfer begins on a time scale of a few tens of fs (the time unit is $1 / J \simeq 20 \mathrm{fs}$, where we have assumed an effective value $J=0.2 \mathrm{eV}$ corresponding to the typical electronic bandwith $W \simeq 0.8 \mathrm{eV}$ of fullerene materials). The coexisting localized and delocalized components both show coherent oscillations caused by the vibronic coupling, and become fully spatially separated after roughly $200 \mathrm{fs}$.
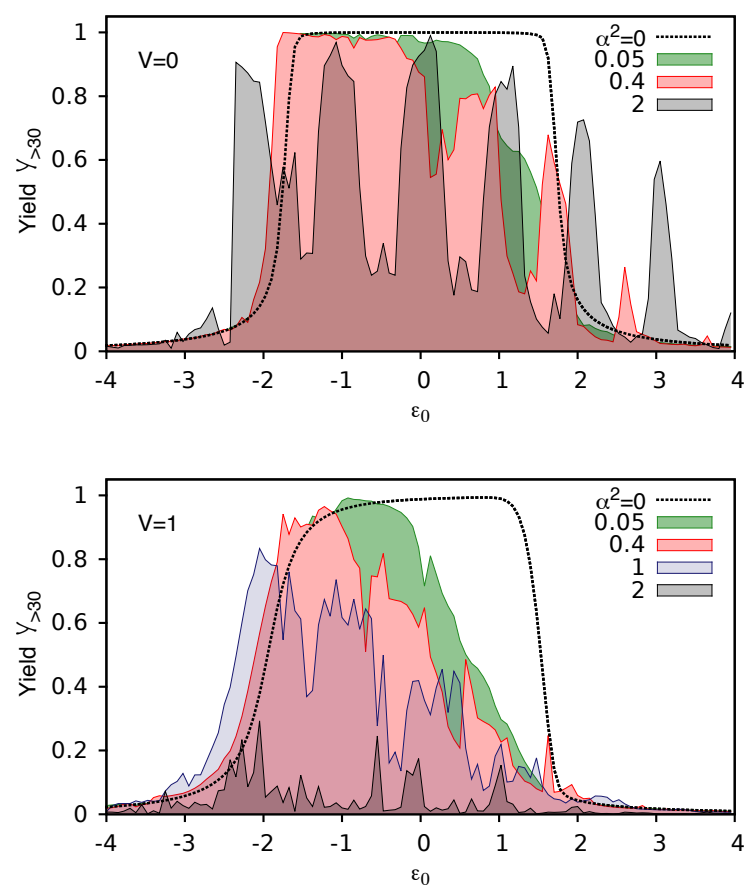

Figure 3: (color online) Yield $\mathcal{Y}_{>30}$ as a function of incoming energy $\epsilon_{0}$, for various values of electron-phonon coupling constant (the non-interacting case $\alpha^{2}=0$ is shown as dashed line). The upper panel is the case without electron-hole binding $(V=0)$, the lower one has $V=1$. The combined effect of Coulomb binding and polaronic dressing of the carriers leads to a strong suppression of the yield.
We now quantify more precisely the degree of charge delocalization, by defining a simple measure of the efficiency for energy transfer in the device. To this aim we consider the site-dependent yield as the total electron probability collected in the long-time limit beyond the site $l=L$ [36:

$$
\mathcal{Y}_{>L}=\lim _{t \rightarrow \infty} \sum_{l>L}\left|A_{l}(t)\right|^{2} .
$$

In practice, we choose $L=30$, which is far enough from the interface and avoids the influence of the interfacial bound states. In addition, $L$ extends beyond the room temperature Coulomb thermal length defined by $L_{T}=$ $V /\left(k_{B} T\right) \simeq 12$ for a typical well of depth $0.3 \mathrm{eV}$, ensuring that charge can be considered as free beyond this range. We also found (for the parameters used here) that a maximal time $t=200$ is sufficient to obtain converged values for $\mathcal{Y}_{>L}$. One must in addition take a chain of about $N=200$ sites in order to prevent the wavepacket from bouncing off the boundary, the portion of the chain $30<l<200$ thus playing the role of a perfect sink.

We start by considering the case without interfacial confinement $(V=0)$ in the upper panel of Fig. 3, scanning for all possible values of the injection energy $\epsilon_{0}$ and changing the strength of the electron-phonon interaction $\alpha^{2}$ (we show in particular $\alpha^{2} \simeq 0.4$, the value corresponding to fullerene molecules [12]). In the non-interacting case, shown for reference, the yield is essentially unity within the bare acceptor bandwidth $-2 J \lesssim \epsilon_{0} \lesssim 2 J$ [21]. A moderate reduction in the yield is observed already at weak electron-phonon coupling, $\alpha^{2}=0.05$, affecting the high energy side of the band (the lower band edge is unaffected, as expected from the existence of long-lived delocalized states at the band bottom [37). Upon increasing $\alpha^{2}$, the dressing of carriers by large molecular deformations leads to the emergence of multiple sub-bands [37] separated in energy by $\omega_{0}$, which reflect the polaronic structure of the acceptor band. The yield is consequently strongly modulated and overall redistributed over a wider injection energy window. We emphasize that for any value of $\alpha^{2}$, however large, one can find energy bands where the yield is close to one, showing that polarons behave as nearly ballistic charge carriers in a homogeneous system (cf. Fig. 2, middle panel).

Bringing in the effect of electron-hole binding (with $V=1$ ) is shown in the lower panel of Fig. 3. While periodic resonances from polaronic dressing are still visible, the global reduction of the yield is clearly dramatic, as could be anticipated from the right panel of Fig. 2. A sharp drop of the overall efficiency occurs with increasing $\alpha^{2}$, not observed in the absence of Coulomb binding. Comparison of the two panels in Fig. 3 shows that the effects of phonon relaxation and interfacial confinement do not simply add up, and that the Coulomb potential has a dramatic interplay with polaronic formation: relaxation processes brought in by the molecular vibrations 
cause a collapse of electronic states towards localized levels near the interface, strongly suppressing the charge delocalization.

The overall photogeneration efficiency of a given device will result from both the efficiency of photon absorption in the required energy range and the ability of excitons to dissociate at the interface. Having addressed the latter process via model Eq. (1), we can conclude that the device performances are strongly suppressed in the presence of strong electron-phonon coupling, indicating that organic molecules with low reorganization energies should be used preferentially. Also, it is apparent from Fig. 3 that high energy electronic states are generally more prone to relaxation, so that the injection of "hotter" carriers should not lead to higher device performances in the presence of vibronic coupling.

In conclusion, we have provided microscopic evidence that the efficiency for energy transfer of electrons across organic interfaces is subtly controlled by an interplay of electrostatic confinement and coherent coupling of electrons to high energy quantized vibrational modes. Our findings rationalize the oscillatory behavior of transferred charges due to molecular vibrations, as recently observed in Refs. [15, 16]. They also reconcile the conflicting views often presented in the literature, arguing in favor of either prominently localized or fully delocalized states [6, 8]. This physics may also be of general relevance to a wider class of light harvesting systems than organic solar cells, designed from inorganic 3] or biological [4 elements, where charge separation occurs also in presence of strong electron-phonon coupling.

We thank X. Blase, A. Salleo and K. Vandewal for useful discussions.

[1] J.-L. Brédas, J. E. Norton, J. Cornil, and V. Coropceanu, Acc. Chem. Res. 42, 1691 (2009).

[2] S. A. Crooker, J. A. Hollingsworth, S. Tretiak, and V. I. Klimov, Phys. Rev. Lett. 89, 186802 (2002).

[3] E. Rozbicki and P. Machnikowski, Phys. Rev. Lett. 100, 027401 (2008).

[4] G. D. Scholes, G. R. Fleming, A. Olaya-Castro, R. van Grondelle, Nat. Chem. 3, 763 (2011).

[5] J. Lee, K. Vandewal, S. R. Yost, M. E. Bahlke, L. Goris, M. A. Baldo, J. V. Manca, and T. Van Voorhis, J. Am. Chem. Soc. (Communication) 132, 11878 (2010).

[6] A. A. Bakulin, A. Rao, V. G. Pavelyev, P. H. M. van Loosdrecht, M. S. Pshenichnikov, D. Niedzialek, J. Cornil, D. Beljonne, and R. H. Friend, Science 335, 1340 (2012).

[7] A. E. Jailaubekov, A. P. Willard, J. R. Tritsch, W-L. Chan, N. Sai, R. Gearba, L. G. Kaake, K. J. Williams, K. Leung, P. J. Rossky and X-Y. Zhu, Nat. Mater. 12, 66 (2013).

[8] S. Gélinas, A. Rao, A. Kumar, S. L. Smith, A. W. Chin, J.
Clark, T. S. van der Poll, G. C. Bazan, and R. H. Friend, Science 343, 512 (2014).

[9] K. Vandewal et al., Nat. Mater. 13, 63 (2014).

[10] A. Kolli, E. J. O'Reilly, G. D. Scholes, and A. OlayaCastro, J. Chem. Phys. 137, 174109 (2012).

[11] V. P. Antropov, O. Gunnarsson, and A. I. Liechtenstein, Phys. Rev. B 48, 7651 (1993).

[12] C. Faber, J. L. Janssen, M. Côté, E. Runge, and X. Blase, Phys. Rev. B 84, 155104 (2011).

[13] V. H. Crespi, J. G. Hou, X.-D. Xiang, M. L. Cohen, and A. Zettl, Phys. Rev. B 46, 12064 (1992)

[14] S. E. Canton et al., Phys. Rev. Lett. 89, 045502 (2002).

[15] S. M. Falke, et al., Science 344, 1001 (2014).

[16] Y. Song, S. N. Clafton, R. D. Pensack, T. W. Kee, and G. D. Scholes, Nat. Commun. 5, 4933 (2014).

[17] D. Feinberg, S. Ciuchi, and F. de Pasquale, Int. J. Mod. Phys. B 4, 1317 (1990).

[18] M. Capone, S. Ciuchi, and C. Grimaldi, Europhys. Lett. 42, 523 (1998).

[19] H. Tamura, J. G. S. Ramon, E. R. Bittner, and I. Burghardt, Phys. Rev. Lett. 100, 107402 (2008).

[20] E. R. Bittner and C. Silva, Nat. Comm. 5, 3119 (2014).

[21] G. Li, A. Nitzan, and M. A. Ratner, Phys. Chem. Chem. Phys. 14, 14270 (2012).

[22] J. Wu, R. J. Silbey, and J. Cao, Phys. Rev. Lett. 110, 200402 (2013).

[23] H. Vázquez and A. Troisi, Phys. Rev. B 88, 205304 (2013).

[24] H. Tamura and I. Burghardt, J. Am. Chem. Soc. (Communication) 135, 16364 (2013).

[25] I. Duchemin and X. Blase, Phys. Rev. B 87, 245412 (2013).

[26] D. Caruso and A. Troisi, PNAS 109, 13498 (2012).

[27] S. L. Smith and A. W. Chin, arXiv:1406.6545.

[28] S. L. Smith and A. W. Chin, Phys. Chem. Chem. Phys. 16, 20305 (2014).

[29] H. Tamura and I. Burghardt, J. Phys. Chem. C 117, 15020 (2013).

[30] Thermally activated hopping, not captured by the present approach, is anyway not relevant in the regime of moderate electron-phonon coupling $\alpha^{2}<2$ studied here, see e.g. [Phys. Rev. Lett. 91256403 (2003)].

[31] P. Kramer and M. Saraceno, "Geometry of the timedependent variational principle in quantum mechanics" (Springer Verlag, Berlin, 1981).

[32] J. Ye, K. Sun, Y. Zhao, Y. Yu, C. K. Lee, and J. Cao, J. Chem. Phys. 136, 245104 (2012).

[33] S. Bera, S. Florens, H. U. Baranger, N. Roch, A. Nazir, and A. W. Chin, Phys. Rev. B 89, 121108(R) (2014).

[34] Y. Yao, L. Duan, Z. Lü, C-Q. Wu, and Y. Zhao, Phys. Rev. E 88, 023303 (2013).

[35] N. Wu, L. Duan, X. Li, and Y. Zhao, J. Chem. Phys. 138, 084111 (2013).

[36] This quantity is related, but not equal, to the internal quantum efficiency commonly extracted from experiments: one can not directly relate the injection energy $\epsilon_{0}$ in Eq. (1) to the incoming photon frequency, because a continuum of possible initial electronic states is generated in the HOMO-LUMO bands upon absorption of light.

[37] S. Ciuchi, F. de Pasquale, S. Fratini, and D. Feinberg, Phys. Rev. B 56, 4494 (1997). 